\title{
Fronteiras da autonomia da gestão local de saúde: inovação, criatividade e tomada de decisão informada por evidências
}

\author{
Boundaries of the autonomy of local health administration: \\ innovation, creativity and evidence-based decision-making
}

Silvio Fernandes da Silva ${ }^{1}$

Nathan Mendes Souza ${ }^{2}$

Jorge Otávio Maia Barreto ${ }^{3}$
${ }^{1}$ Instituto Sírio Libanês de Ensino e Pesquisa. R. Coronel Nicolau dos Santos 69, Bela Vista. 01308060 São Paulo SP Brasil. fernandessilvio90@gmail. com

${ }^{2}$ Departamento de Ciência e Tecnologia, Secretaria de Ciência, Tecnologia e Insumos Estratégicos, Ministério da Saúde.

${ }^{3}$ Programa de PósGraduação em Políticas Públicas, Universidade Federal do Piauí.
Abstract The scope of this article was to identify the boundaries of the autonomy of local administration in the context of the federal pact in the Brazilian Unified Health System and the importance and potential for promoting innovation, creativity and evidence-based decision-making by local governments. The methodology used was to ask questions that favored dialogue with the specific literature to identify the influence of centrally-formulated policies in spaces of local autonomy and then to identify strategies to foster innovation, creativity and the systematic use of evidence-based research in health policy implementation. A gradual reduction in municipal decision-making autonomy was detected due to increased financial commitment of the municipalities resulting from responsibilities assumed, albeit with the possibility of reverting this trend in the more recent context. Some determinants and challenges for the dissemination of innovative practices were analyzed and some relevant national and international experiences in this respect were presented. The conclusion drawn is that it is possible to make local decision-making more effective provided that initiatives are consolidated to promote this culture and the formulation and implementation of evidence-based health policies. Key words Decentralization, Local government, Health systems, Innovation, Evidence in health
Resumo O propósito deste artigo foi identificar as fronteiras da autonomia da gestão municipal no contexto do pacto federativo do Sistema Único de Saúde brasileiro e a importância e possibilidade de fomentar inovação, criatividade e tomada de decisão, informada pelas melhores evidências pelos governos locais. Metodologicamente partimos de questões que propiciaram diálogo com a literatura específica, visando identificar a influência das políticas com formulação centralizada nos espaços de autonomia local para, em seguida, identificar estratégias de incentivo à inovação, criatividade e uso sistemático da evidência de pesquisas na implementação de políticas em saúde. Constatamos uma redução gradativa da autonomia decisória pelo maior comprometimento financeiro dos municípios, decorrente das novas responsabilidades assumidas, bem como a possibilidade de reversão dessa tendência no contexto mais recente. Analisamos também determinantes e desafios para a difusão de práticas inovadoras e apresentamos experiências nacionais e internacionais com essa finalidade. Concluímos ser possível tornar mais efetivo o processo decisório local, desde que se consolidem iniciativas que estimulem essa cultura e a formulação e implementação de políticas informadas por evidências.

Palavras-chave Descentralização, Governo local, Sistemas de saúde, Inovação, Evidências em saúde 


\section{Introdução}

O processo de descentralização no Sistema Único de Saúde contribuiu para a expansão progressiva dos sistemas municipais de saúde. Praticamente inexistentes na década de 1960, estes sistemas se ampliaram muito nas últimas décadas. A porcentagem de hospitais públicos sob gestão municipal em 2005 era de 25\% e, em fevereiro de 2013, atingiu 52\%, resultando um aumento de 49.646 para 89.728 de leitos de internação hospitalar administrados pelos municípios neste período ${ }^{1}$.

A transferência na gestão ocorreu também em unidades de atenção não hospitalar e, em 2013, dos 70.441 estabelecimentos públicos cadastrados no Cadastro Nacional de Estabelecimentos de Saúde (CNES), 67.014 (95,1\%) eram administrados pelos municípios ${ }^{2}$. Além disso, programas assistenciais e estratégias de mudança nos modelos de saúde, cuja coordenação cabe aos municípios, cresceram exponencialmente. Os agentes comunitários de saúde que eram 59.066 e as equipes de saúde da família 2.054, em janeiro de 1988, passaram para 253.140 e $33.193 \mathrm{em}$ março de 2013, respectivamente 3 .

A municipalização da saúde teve como causa importante a indução por parte do governo federal pela criação de incentivos financeiros que estimula os municípios a assumirem novas responsabilidades na gestão e na prestação de ações de saúde. Se no início desse processo as gestões semiplenas, previstas em norma operacional publicada em 1993 (NOB/SUS 93) , propunham regras mais flexíveis para a transferência de recursos federais e garantiam razoável autonomia de decisão para os governos municipais, a partir de 1996 (NOB/SUS 96) ${ }^{5}$ essa flexibilidade diminuiu; aumentou a vinculação do repasse à adesão às diretrizes formuladas centralizadamente, que reduziu a fronteira de autonomia decisória local.

Com a publicação do Pacto pela Saúde ${ }^{6}$, em 2006, e do Decreto $7508^{7}$, em 2011, identifica-se a possível reversão dessa tendência. $O$ processo de pactuação de políticas proposto por esses instrumentos, especialmente os relacionados ao objetivo de promover maior integração de serviços e regionalização do SUS, tende a ampliar o protagonismo dos governos locais e isso poderá resultar em aumento de autonomia decisória.

Essa autonomia é fundamental para a inovação e criatividade municipal, ajustando os modelos de gestão e atenção à saúde aos contextos locais e às necessidades singulares de suas regiões e comunidades. Essa é uma das condições da universalidade do acesso aos serviços de saúde e melhoria da qualidade da atenção. A outra diz respeito a auxiliar os gestores municipais a trilharem esse caminho informando a tomada de decisões pelas melhores evidências.

Partindo do pressuposto de que deve existir coerência entre os espaços de autonomia dos governos locais e o escopo de decisões atribuível a eles pelas políticas de saúde, indagamos: É possível delimitar fronteiras e espaços de autonomia dos governos locais no atual contexto de implementação do SUS? Quais as melhores alternativas para qualificar o processo de decisão local, fomentando a utilização das melhores evidências com inovação e criatividade?

Metodologicamente, em busca de resposta para essas questões, inicialmente exploramos aspectos da descentralização na literatura acerca do assunto. Dialogamos essa discussão com os atuais dispositivos normativos e políticos do SUS. Com isso, analisamos a descentralização no que se refere ao caráter indutor das políticas com formulação centralizada e sua influência nas fronteiras da autonomia dos governos locais. Em seguida identificamos ideias e exemplos de como promover a cultura de inovação e criatividade como fator de otimização do potencial da gestão local e a importância do uso de evidências de pesquisa na tomada de decisão.

\section{Descentralização, indução central e fronteiras da autonomia}

A descentralização da saúde teve como marco o artigo 198 da Constituição Federal ${ }^{8}$, regulamentado pelas leis $8.080^{9}$ e $8.142^{10}$, que estabeleceram que o SUS deve ser organizado de forma descentralizada, "com direção única em cada esfera de governo". As normas operacionais da década de 1990 tornaram a municipalização o elemento mais importante do processo de descentralização no SUS.

Este processo sofreu influência de interesses diversos, podendo-se destacar dois como os mais importantes. Em um deles, a transferência de responsabilidades para os municípios, defendida pelo movimento da reforma sanitária, pode ser considerada como uma 'bandeira de luta' para democratizar o processo decisório e imprimir maior racionalidade e eficiência na implementação das políticas públicas de saúde. O outro resulta das políticas de ajuste fiscal da década de 1990, determinadas pela agenda neoliberal do governo, que atribuíam às políticas de descentralização potencial para reduzir gastos, ao mesmo tempo em que se exercia forte controle fiscal ${ }^{11,12}$. 
Esses dois diferentes interesses e racionalidades da descentralização das políticas sociais são destacados por Silva ${ }^{13}$ :

Enquanto em uma das dimensões predomina a lógica de descentralização como uma proposta política de deslocamento dos eixos de poder para as instâncias locais, ampliando, como consequência, os mecanismos institucionais de democracia formal e promovendo a participação direta da sociedade, na outra predomina a lógica da racionalidade econômica, onde se descentraliza para cobrar maior racionalidade e eficiência na execução das políticas setoriais.

Com a descentralização, os gastos municipais cresceram gradativamente, passando de $7 \%$ do total nacional gasto no SUS em 1980, para $28 \%$, em $2010^{14}$. Um dos principais fatores para isso foi a necessidade de complementar os repasses de custeio federal, e se deveu especialmente a gastos com pessoal. O número de postos de trabalho em saúde nos municípios cresceu de 47.038 (17,6\% do total do país) em 1980 para 997.137 (68,8\% do total existente)em $2005^{15}$.

As frequentes dificuldades orçamentárias e financeiras, decorrentes do crescente gasto municipal com saúde (a maioria dos municípios ultrapassa o limite mínimo de $15 \%$ da receita estabelecido pela Emenda Constitucional 29), podem interferir na autonomia local para inovações na gestão. Além disso, a vinculação do repasse de recursos federais ao cumprimento de regras pré-determinadas também pode funcionar como fator que limita a inovação e criatividade no âmbito da gestão municipal. A partir de 2006, com a implementação do Pacto pela Saúde, as centenas de formas vinculadas de repasse de recursos financeiros federais, instituídas especialmente após a NOB/SUS 96, foram reduzidas a cinco blocos de financiamento, ampliando a margem de manobra no uso dos recursos e amenizando um pouco o problema ${ }^{16}$.

Diante desses dilemas, maior equilíbrio nas relações interfederativas para orientar o processo de descentralização, mostra-se essencial. Analisando essa questão, Arretche ${ }^{17}$ entende ser necessário harmonizar indução, regulação e autonomia. 'Indução' tendo como principal objetivo promover uniformidade na implementação das políticas públicas no território nacional. 'Regulação', para buscar maior eficiência e transparência na aplicação dos recursos públicos, pois não há garantia intrínseca de que os governantes locais sempre usarão os recursos adequadamente, de forma responsável e comprometida com o interesse do cidadão. 'Autonomia' como condição indispensável para que se expressem inovação e criatividade na execução das ações de saúde.

Nesse contexto, como demarcar as fronteiras de autonomia decisória local? Não pretendemos estabelecer limites precisos para isso e sim propiciar uma aproximação que permita a identificação de espaços onde a inovação e criatividade local possam ser praticadas, alcançando os benefícios que o conhecimento e as evidências científicas podem proporcionar ao processo de tomada de decisão na política de saúde.

Nas regras atuais, implantadas após a vigência do Pacto pela Saúde, os municípios decidem, no processo de pactuação, quais serviços localizados no território municipal ficarão sob sua gestão. O decreto $7.508^{7}$, de 2011, introduz o Contrato Organizativo de Ação Pública (COAP) como instrumento para demarcar responsabilidades e atribuições nas relações interfederativas. Além disso, devem ser considerados como legados indutores, com influência na delimitação da fronteira de autonomia decisória local, os seguintes dispositivos normativos e políticos do SUS: a Política Nacional de Atenção Básica (PNAB) ${ }^{18}$; o Programa Nacional de Melhoria do Acesso e da Qualidade da Atenção Básica (PMAQ-AB) ${ }^{19}$; o Programa de Valorização do Profissional da Atenção Básica (PROVAB) ${ }^{20}$; a Política Nacional de Regulação do SUS ${ }^{21}$ e as Diretrizes para organização das redes de atenção à saúde no âmbito do SUS ${ }^{22}$. O Quadro 1 apresenta as características dessas políticas, quanto aos seus objetivos principais, estratégias de indução, mecanismos de regulação e espaços de autonomia local.

$\mathrm{Na}$ análise do Quadro 1 pode-se verificar que os mecanismos indutórios se concretizam pela transferência de recursos financeiros e também por disseminar aspectos teórico-conceituais e diretrizes nacionais estratégicas e operacionais para implementação das políticas. Essas informações fornecem pistas para delimitar as fronteiras de autonomia local. Uma área em que o protagonismo dos municípios para implementação do SUS é mais acentuado é a Atenção Primária em Saúde (APS), que traz desafios para a agenda decisória local que podem ser situados em duas dimensões principais, proposta na Política $\mathrm{Na}$ cional de Atenção Básica (PNAB): coordenar o cuidado integral e ordenar a longitudinalidade do cuidado, na perspectiva de sua continuidade em redes regionalizadas de atenção à saúde.

Com relação à primeira dimensão, em uma análise mais pormenorizada das políticas analisadas, a agenda local se depara com os seguintes assuntos: (i) integrar ações programáticas 
Quadro 1. Aspectos da indução, regulação e autonomia local de políticas do SUS.

\begin{tabular}{|c|c|c|c|c|c|c|}
\hline \multirow[b]{2}{*}{ Atributos } & \multicolumn{6}{|c|}{ Políticas } \\
\hline & Regulação & COAP & PMAQ-AB & PROVAB & PNAB & Redes \\
\hline $\begin{array}{c}\text { Marco de } \\
\text { implantação }\end{array}$ & $\begin{array}{l}\text { Portaria do } \\
\text { MS n }{ }^{\circ} 1559 \text {, de } \\
01 / 08 / 2008^{19}\end{array}$ & $\begin{array}{l}\text { Decreto } \\
\text { Presidencial no } \\
7508 \text { 28/06/2011 }\end{array}$ & $\begin{array}{l}\text { Portaria do MS } \\
\text { no } 1654 \text {, de } \\
19 / 07 / 20111^{7}\end{array}$ & $\begin{array}{l}\text { Portaria } \\
\text { interministerial } \\
\text { no } 2087 \text {, de } \\
01 / 09 / 2011^{18}\end{array}$ & $\begin{array}{l}\text { Diretriz } \\
\text { orientadora do MS } \\
\text { de } 2012^{16}\end{array}$ & $\begin{array}{l}\text { Portaria do MS } \\
\text { no } 4279 \text {, de } \\
30 / 12 / 2012^{20}\end{array}$ \\
\hline $\begin{array}{l}\text { Objetivos } \\
\text { principais }\end{array}$ & $\begin{array}{l}\text { Implantar } \\
\text { as ações de } \\
\text { regulação de } \\
\text { sistemas, da } \\
\text { atenção e do } \\
\text { acesso à saúde }\end{array}$ & $\begin{array}{l}\text { Regulamentar a } \\
\text { Lei no } 8.080 ; \\
\text { Organizar o } \\
\text { planejamento do } \\
\text { SUS; Promover } \\
\text { articulação } \\
\text { interfederativa }\end{array}$ & $\begin{array}{l}\text { Induzir } \\
\text { ampliação do } \\
\text { acesso e melhoria } \\
\text { da qualidade da } \\
\text { APS; Promover } \\
\text { cultura de } \\
\text { avaliação, } \\
\text { monitoramento e } \\
\text { negociação }\end{array}$ & $\begin{array}{l}\text { Valorizar o } \\
\text { profissional de } \\
\text { saúde que atue } \\
\text { na APS e da } \\
\text { Estratégia Saúde } \\
\text { da Família }\end{array}$ & $\begin{array}{l}\text { Ampliar } \\
\text { resolutividade da } \\
\text { APS; Coordenar o } \\
\text { cuidado integral; } \\
\text { Ordenar a } \\
\text { longitudinalidade } \\
\text { do cuidado em } \\
\text { rede }\end{array}$ & $\begin{array}{l}\text { Estabelecer as } \\
\text { diretrizes de } \\
\text { organização } \\
\text { das redes de } \\
\text { atenção à } \\
\text { saúde no SUS }\end{array}$ \\
\hline $\begin{array}{l}\text { Estratégias } \\
\text { de indução }\end{array}$ & $\begin{array}{l}\text { Repasse de } \\
\text { recursos } \\
\text { financeiros para } \\
\text { investimento } \\
\text { e custeio dos } \\
\text { complexos } \\
\text { reguladores }\end{array}$ & $\begin{array}{l}\text { Diretrizes } \\
\text { prioritárias } \\
\text { nacional e } \\
\text { estadual de saúde; } \\
\text { Incentivo } \\
\text { financeiro pelo } \\
\text { cumprimento de } \\
\text { metas }\end{array}$ & $\begin{array}{l}\text { Implementar } \\
\text { diretrizes, } \\
\text { avaliação, } \\
\text { monitoramento, } \\
\text { educação } \\
\text { permanente } \\
\text { e apoio } \\
\text { institucional; } \\
\text { Incentivos } \\
\text { financeiros } \\
\text { para adesão e } \\
\text { cumprimento de } \\
\text { metas }\end{array}$ & $\begin{array}{l}\text { Aos profissionais: } \\
\text { curso de } \\
\text { especialização } \\
\text { e bônus para } \\
\text { provas de } \\
\text { residência } \\
\text { médica; } \\
\text { Aos municípios: } \\
\text { incentivos } \\
\text { financeiros para } \\
\text { custeio }\end{array}$ & $\begin{array}{l}\text { Piso de Atenção } \\
\text { Básica (PAB) } \\
\text { fixo; Incentivos } \\
\text { adicionais } \\
\text { proporcionalmente } \\
\text { expressivos } \\
\text { para Saúde da } \\
\text { Família, Agente } \\
\text { Comunitário de } \\
\text { Saúde e Saúde } \\
\text { Bucal (PAB } \\
\text { variável) }\end{array}$ & $\begin{array}{l}\text { Diretrizes } \\
\text { conceituais, } \\
\text { estratégicas e } \\
\text { operacionais } \\
\text { para todos os } \\
\text { componentes } \\
\text { do SUS }\end{array}$ \\
\hline $\begin{array}{l}\text { Mecanismos } \\
\text { de regulação }\end{array}$ & $\begin{array}{l}\text { Garantia de } \\
\text { execução de } \\
\text { processos (ex.: } \\
\text { cadastros, } \\
\text { contratos, PPI, } \\
\text { protocolos) }\end{array}$ & $\begin{array}{l}\text { Controle pelos } \\
\text { órgãos interno } \\
\text { e externo do } \\
\text { MS em caso de } \\
\text { descumprimento } \\
\text { de } \\
\text { responsabilidades }\end{array}$ & $\begin{array}{l}\text { Mecanismos de } \\
\text { avaliação externa } \\
\text { periódica }\end{array}$ & $\begin{array}{l}\text { Obediência aos } \\
\text { requisitos legais } \\
\text { de contratação e } \\
\text { provimento de } \\
\text { moradia }\end{array}$ & $\begin{array}{l}\text { Obediência às } \\
\text { diretrizes gerais da } \\
\text { PNAB; Controle } \\
\text { por órgãos interno } \\
\text { e externo do } \\
\text { MS em caso de } \\
\text { descumprimento } \\
\text { de } \\
\text { responsabilidades }\end{array}$ & $\begin{array}{l}\text { Difusa nas } \\
\text { demais } \\
\text { políticas }\end{array}$ \\
\hline $\begin{array}{l}\text { Espaços de } \\
\text { autonomia } \\
\text { local }\end{array}$ & $\begin{array}{l}\text { Gestão ou } \\
\text { cogestão de } \\
\text { complexos } \\
\text { reguladores; } \\
\text { Elaboração } \\
\text { de protocolos } \\
\text { clínicos e de } \\
\text { regulação; } \\
\text { Contratualização } \\
\text { com prestadores } \\
\text { de serviços }\end{array}$ & $\begin{array}{l}\text { Planejamento } \\
\text { ascendente } \\
\text { considerando as } \\
\text { necessidades do } \\
\text { nível local }\end{array}$ & $\begin{array}{l}\text { Responsabilidade } \\
\text { pela } \\
\text { implementação } \\
\text { de estratégias } \\
\text { de avaliação, } \\
\text { de processos } \\
\text { educacionais } \\
\text { e de apoio } \\
\text { institucional } \\
\text { visando atender } \\
\text { as diretrizes } \\
\text { nacionais }\end{array}$ & $\begin{array}{l}\text { Inerentes à } \\
\text { inserção dos } \\
\text { profissionais na } \\
\text { APS (cuidado } \\
\text { multiprofissional, } \\
\text { integral, } \\
\text { longitudinal, } \\
\text { contextualizado e } \\
\text { coordenador dos } \\
\text { serviços) }\end{array}$ & $\begin{array}{l}\text { Aplicar estratégias } \\
\text { adicionais que } \\
\text { não contrariem as } \\
\text { diretrizes nacionais } \\
\text { (programar ações } \\
\text { de acordo com } \\
\text { as necessidades; } \\
\text { instituir avaliação; } \\
\text { promover ações } \\
\text { educacionais) }\end{array}$ & $\begin{array}{l}\text { Participação } \\
\text { no } \\
\text { planejamento, } \\
\text { gerenciamento } \\
\text { e governança } \\
\text { das redes, no } \\
\text { componente } \\
\text { municipal }\end{array}$ \\
\hline
\end{tabular}

Fonte: Ministério da Saúde, Brasil. 
e demanda espontânea; (ii) articular ações de prevenção, promoção, tratamento e reabilitação; (iii) articular trabalho multiprofissional e interdisciplinar das equipes de saúde; (iv) direcionar o processo de trabalho de forma usuário-centrado; (v) desenvolver ações de educação permanente em saúde; (vi) estimular a participação do usuário visando o autocuidado em saúde; (vii) definir estratégias de institucionalização da avaliação da atenção básica; (viii) instituir acolhimento humanizado e priorizando o acesso baseado em risco epidemiológico e social.

Em relação à segunda: (i) promover resolutividade da APS, identificando riscos, necessidades e demandas; (ii) utilizar e articular diferentes tecnologias de cuidado individual e coletivo visando à prática de uma clínica ampliada que considere o usuário em suas necessidades singulares; (iii) elaborar, acompanhar e gerir projetos terapêuticos singulares; (iv) acompanhar e organizar o fluxo dos usuários entre os pontos de atenção das redes de atenção à saúde; (v) articular ações intersetoriais, comunitárias e sociais; (vi) incorporar tecnologias, ferramentas e dispositivos de gestão da clínica na gestão do cuidado; (vii) alimentar sistemas e analisar informações em saúde; (viii) desenvolver cultura de negociação e contratualização; (ix) regular o acesso aos demais níveis de atenção.

Esses desafios situam-se nos espaços de autonomia local. Enfrentá-los traz para os tomadores de decisão a necessidade de analisar adequadamente o contexto e identificar fragilidades e oportunidades a serem exploradas. Traz, fundamentalmente, o desafio de serem inovadores e criativos, procurando tomar as decisões orientadas pelas melhores evidências, temas que serão tratados nos tópicos seguintes.

\section{Inovação e criatividade na gestão local de saúde}

A saúde é um campo fértil para inovações informadas por evidências, mas, mesmo quando estas são bem sucedidas em um local, leva tempo para essas novas ideias e práticas se difundirem, levando seus benefícios para todo o sistema. Isso é verdadeiro em relação a produtos inovadores, mas para as tecnologias organizacionais complexas, como novas formas de atribuição de tarefas (i.e.: task shiffting), mudanças nos processos de trabalho ou modalidades alternativo/combinadas de remuneração (i.e.: pay-for-performance), a disseminação da inovação no sistema de saúde pode levar ainda mais tempo.
A difusão das práticas inovadoras é, portanto, um grande desafio e a taxa de difusão da inovação em uma organização/sistema é condicionada por fatores complexos como as percepções individuais e coletivas sobre a inovação, características dos indivíduos e instituições que podem adotar a mudança e fatores contextuais e políticos de uma organização/sistema. No entanto, algumas estratégias podem acelerar a taxa de difusão de inovações dentro de sistemas e organizações de saúde, tais como: dar visibilidade às inovações, descobrir e apoiar experiências inovadoras, investir em early adopters, confiar e permitir a recombinação de ideias no âmbito da implementação, incentivar ambientes institucionais favoráveis à mudança e liderar pelo exemplo ${ }^{23}$.

No Brasil, a distribuição de competências no sistema de saúde remete às organizações locais, a implementação dos programas e ações, em geral mediante a distribuição de recursos financeiros federais, configurando mecanismo de incentivo baseado na concentração orçamentária e de formulação das políticas e desconcentração de recursos para implementação em nível local. Nesse contexto, indaga-se sobre limites e possibilidades para favorecer a inovação no SUS, especialmente para incentivar seu surgimento e disseminação.

No SUS, podem-se apontar exemplos de inovações nacionalmente disseminadas, mas com implementação no contexto local/regional e endereçadas a diferentes níveis do sistema de saúde. Por exemplo, na esfera da gestão, os contratos organizativos de ação pública da saúde (COAP) e os novos modelos institucionais, como as fundações estatais, representam novas formas de organização com importantes repercussões sobre a dinâmica de accountability. No âmbito do financiamento, observa-se a ascensão e disseminação em todo o país do pagamento por resultados, após o desenvolvimento do que pode ser o maior esquema de pay-for-performance do mundo na APS, o Programa Nacional de Melhoria do Acesso e da Qualidade (PMAQ). Novos modelos de provisão de cuidados estão se conformando em redes assistenciais regionalizadas e coordenadas pela APS, para superar a histórica segmentação/ fragmentação dos níveis assistenciais no acesso aos serviços de saúde. Além disso, registram-se importantes iniciativas de incentivo à inovação mediante premiações institucionais, como o Prêmio de Incentivo em Ciência e Tecnologia para o SUS e o Prêmio Nacional de Incentivo à Promoção do Uso Racional de Medicamentos, ambos promovidos pelo Ministério da Saúde, através da Secretaria de Ciência, Tecnologia e Insumos 
Estratégicos (SCTIE), e capacitações/treinamentos focados no desenvolvimento de competências úteis aos processos inovadores, como as oficinas para a utilização das Ferramentas SUPPORT para Políticas Informadas por Evidências, realizadas pela EVIPNet Brasil.

Especialmente no campo da promoção da inovação na gestão pública da saúde, exemplo de iniciativa é o Portal de Inovação na Gestão do SUS, uma ferramenta de gestão do conhecimento, idealizada e mantida pela parceria institucional entre a Organização Pan-Americana da Saúde, Ministério da Saúde, Conselho Nacional de Secretarias Municipais de Saúde (CONASEMS) e Conselho Nacionais de Secretários Estaduais de Saúde (CONASS). O portal visa apoiar gestores, profissionais, dirigentes e quadros técnicos do SUS e da saúde suplementar para o acesso aos principais serviços informativos e de gestão de conhecimento e desenvolver uma 'comunidade de prática' entre gestores, criando um ambiente colaborativo de socialização de conhecimento e experiências.

No âmbito da gestão municipal, a 'folga' para inovação parece se acomodar nos processos de implementação das políticas de saúde, impulsionada especialmente pela criatividade de gestores e trabalhadores do sistema de saúde, mas com elevado potencial de inserção da sociedade civil. Pode-se citar como exemplos de clima institucional propício à criatividade iniciativas para promover o engajamento construtivo dos usuários e profissionais de saúde, mediante a criação de mecanismos de interação entre governo e sociedade e reforçar a eficiência governamental e transparência com o apoio da sociedade civil ${ }^{24}$; geração e uso de novos indicadores de monitoramento e avaliação de processos e resultados e concepção e uso de novas metas para a gestão, a exemplo dos Objetivos de Desenvolvimento do Milênio $(\mathrm{ODM})^{25,26}$, como fatores que favorecem ambientes para o surgimento e crescimento da inovação na gestão local do SUS.

Nesse ponto é relevante o papel da pesquisa como estratégia para promover e evidenciar a inovação e criatividade no SUS. O Brasil experimenta o aumento dos custos e gastos em saúde em decorrência do envelhecimento populacional e da alta carga de doenças infecto-parasitárias concomitantes às doenças crônicas não transmissíveis, assim como o aumento da expectativa e das demandas dos usuários em relação aos serviços e sistemas de saúde no Brasil, daí a importância das iniciativas para sintetizar e traduzir os achados dessas pesquisas e transformá-las em ações efetivas a custos apropriados.
Devido à grande variabilidade de cenários, o Brasil tem adotado incentivos à produção de conhecimento descentralizada, adaptada aos diferentes caracteres geográficos e socioeconômicos. Exemplo importante é a Pesquisa para o SUS (PPSUS), que financia pesquisas sobre temas prioritários de saúde com o intuito de fortalecer as capacidades locais de investigação. Essa modalidade de fomento descentralizado tem como objetivo reduzir desigualdades regionais na produção do conhecimento científico e fortalecer a capacidade local da gestão, especialmente para lidar com problemas de saúde e com a produção de conhecimento científico como subsídio de enfrentamento e decisão $0^{27}$.

No entanto, é preciso reconhecer que os resultados concretos de intervenções para desencadear a inovação nos cuidados de saúde, dependem de processos complexos e variáveis que incluem a interação entre a inovação e o contexto da sua introdução, mas também da sustentabilidade institucional das iniciativas, condição sine qua non dos processos de inovação criativos e relevantes para a maioria da população brasileira no âmbito da saúde. A inovação, portanto, adapta-se aos contextos organizacionais. E o clima receptivo para a inovação só pode ser desenvolvido ao longo do tempo, assegurando a longitudinalidade das iniciativas, enquanto a criatividade e a construção de 'espaços' institucionais para seu exercício podem ser apontados como determinantes para isso.

\section{Sistemas locais de saúde informados por evidências}

O SUS brasileiro é mundialmente conhecido por objetivar a universalização da promoção e cuidados à saúde de seus cidadãos, sob a égide da integralidade e equidade ${ }^{28,29}$. Procura fazê-lo, através de princípios altruístas, com gestão, financiamento e provisão de serviços públicos e privados (saúde suplementar), visando dirimir persistentes desigualdades na longevidade e qualidade de vida de brasileiros pertencentes a diferentes extratos socioeconômicos e raciais. Complementando o caráter indutor central das políticas de saúde com o exercício da inovação e criativa autonomia, governos loco-regionais poderão gerar novos ciclos evolutivos de seus sistemas de saúde. Entretanto, a aplicação do conhecimento produzido pela pesquisa científica, a disponibilidade de informações atualizadas e fidedignas e a observância dos valores das pessoas afetadas/interessadas e marcos legais existentes, 
ainda não são ideias amplamente disseminadas para tornar os serviços e sistemas de saúde mais efetivos e sustentáveis.

Nesse sentido, a 58 a Assembléia Mundial da Saúde $(2005)^{30}$, a Agenda de Saúde para as Américas 2008-17 da Organização Pan-Americana de Saúde ${ }^{31}$ e o relatório mundial da saúde da OMS de 2012, intitulado No Health Without Research ${ }^{32}$ constituem exemplos internacionais de recomendações para que as políticas, sistemas e serviços de saúde sejam mais informados pelas melhores evidências, conhecimentos e valores sociais existentes.

Os governos da província de Ontário (Canadá) e da Austrália são jurisdições que inseriram planos de 'tradução de conhecimento' (knowledge translation) em suas agendas decisórias. O Ministério da Saúde de Ontário assinou em abril de 2013 plano de tradução de conhecimento de CAN\$ 4.9 milhões com a Universidade de McMaster, visando a produção de evidência de pesquisa; promoção e apoio ao uso das melhores evidências globais e provinciais relacionadas a sistemas de saúde; apoio ao desenvolvimento de políticas para aumentar o desempenho e a sustentabilidade do sistema de saúde provincial ${ }^{33}$.

Semelhantemente, o Ministério da Saúde australiano publicou, em fevereiro de 2013, o relatório Strategic Review of Health and Medical Research in Australia - Better Health Through Research $^{34}$ estabelecendo recomendações e verbas para incrementar pesquisas e o uso de seus resultados nos sistemas de saúde com foco na tradução do conhecimento e seu impacto, monitoramento dos investimentos e resultados; inserção da pesquisa em todos os níveis dos sistemas de saúde; desenvolvimento de capacidade de pesquisa, participação e treinamento em pesquisa por parte dos profissionais de saúde; estabelecimento e retenção desse capital humano de pesquisa nos serviços de saúde; apoio a pesquisa direcionada às necessidades prioritárias dos sistemas de saúde; aceleramento do sistema de inovação em saúde e fortalecimento de serviços e sistemas de saúde informados por evidências.

No Brasil, a Rede de Políticas Informadas por Evidências (EVIPNet Brasil: http://brasil.evipnet. org/) alinha-se aos esforços globais para conectar evidências de pesquisa às ações em saúde. A Evipnet Brasil é apoiada pela Organização Mundial de Saúde (OMS), Organização Pan-Americana da Saúde do Brasil (OPAS), Centro Latino-Americano e do Caribe de Informação em Ciências da Saúde (Bireme) e financiada pelo Ministério da Saúde (MS), através do seu Departamento de Ci- ência e Tecnologia (DECIT). Esta rede busca fortalecer os sistemas de saúde através da elaboração de políticas informadas por evidências científicas de qualidade.

Essa rede vem desenvolvendo diversas atividades desde sua criação, em 2007, tais como: 1) estabelecimento de parcerias entre gestores, acadêmicos e a sociedade civil organizada, sendo a colaboração institucional entre o DECIT/SCTIE/ MS, a Bireme/Opas e o CONASEMS o exemplo mais prolífico; 2) desenvolvimento do Portal EVIPNet Brasil; 3) produção de sínteses de evidências para a políticas (Policy Briefs ou Evidence Briefs for Policy $)^{35}$, para o enfrentamento de problemas prioritários de saúde como mortalidade perinatal e materna, crack e hipovitaminose A; 4) capacitações das coordenações das áreas técnicas de avaliação de tecnologia da saúde, alimentação e nutrição, saúde mental, saúde da criança e saúde da mulher ${ }^{34}$, seja através de atividades de educação continuada com técnicos dessas áreas no MS, realizações de oficinas em congressos nacionais $^{34}$, ou em oficinas internacionais independentes. Essas capacitações buscam sempre promover também o intercâmbio entre múltiplos atores sociais ${ }^{35,36}$.

Dentre as equipes nacionais da EVIPNet Global, a equipe brasileira foi pioneira ao inovar com a criação, em 2010, do Núcleo de Evidências em Saúde/Estação Biblioteca Virtual em Saúde (NEv/ EBVS), no município de Piripiri (PI) ${ }^{37}$ (Quadro 2). O NEv/EBVS Piripiri foi instituído por decreto municipal e formado por profissionais efetivos da SMS, os quais foram capacitados para o uso das Ferramentas SUPPORT (SUPPORT Tools for evidence-informed health Policymaking) de elaboração de políticas de saúde informadas por evidências ${ }^{38,39}$, Saúde Baseada em Evidências e Avaliação de Tecnologias em Saúde. A experiência foi desenvolvida e monitorada no período março de 2010 a dezembro de 2012, e foi apoiada pela EVIPNet Brasil, BIREME, MS e governo municipal. A descentralização do financiamento e dos cuidados primários de saúde, a premência do uso de evidências na gestão, a presença de forte liderança, a instituição de marco legal da iniciativa ${ }^{40} \mathrm{e}$ o favorável contexto político local explicam o exitoso desenvolvimento e implementação do NEv/ EBVS de Piripiri.

As atividades da plataforma de tradução de conhecimento NEv/EBVS Piripiri consistiram na: 1) capacitação de recursos humanos locais na aquisição, avaliação, adaptação e aplicação das melhores evidência globais e locais; 2) produção de sínteses de evidências cientificas para políti- 
Quadro 2. Apresentação da Rede de Políticas Informadas por Evidências (EVIPNet Brasil) e do Núcleo de Evidência em Saúde (NEv) de Piripiri.

\begin{tabular}{|c|c|c|}
\hline Atributos & EVIPNet Brasil & Núcleo de Evidência em Saúde (NEv) de Piripiri \\
\hline Data de criação & Julho de 2007 & Março de 2010 \\
\hline Objetivos & $\begin{array}{l}\text { Estabelecer mecanismos que facilitem } \\
\text { o uso de evidências de pesquisa na } \\
\text { formulação e implementação de políticas } \\
\text { de saúde e nos processos de tomada de } \\
\text { decisão, nos níveis de gestão dos serviços } \\
\text { e sistemas de saúde; }\end{array}$ & $\begin{array}{l}\text { Democratizar o acesso às fontes de informação; } \\
\text { Promover inclusão digital através do acesso às } \\
\text { fontes de informação científica e técnica em } \\
\text { saúde; Promover disseminação de evidências } \\
\text { em saúde no âmbito local junto a gestores, } \\
\text { profissionais de saúde e sociedade; }\end{array}$ \\
\hline Missão & $\begin{array}{l}\text { Apoiar a institucionalização do uso } \\
\text { sistemático da evidência de pesquisa para } \\
\text { informar a formulação e implementação } \\
\text { de políticas, programas e serviços de } \\
\text { saúde no âmbito do SUS. }\end{array}$ & $\begin{array}{l}\text { Apoiar a gestão da saúde local no processo } \\
\text { de tomada de decisão sobre formulação } \\
\text { e implementação de políticas de saúde, } \\
\text { disponibilizando as melhores evidências } \\
\text { científicas aos gestores, trabalhadores e } \\
\text { sociedade civil. }\end{array}$ \\
\hline Governança & $\begin{array}{l}\text { (EVIPNet) Brasil é subsidiária da } \\
\text { EVIPNet: Evidence-informed Policy } \\
\text { Network, da Organização Mundial da } \\
\text { Saúde e Organização Pan-Americana da } \\
\text { Saúde. Secretaria Executiva situada no } \\
\text { Departamento de Ciência e Tecnologia } \\
\text { da Secretaria de Ciência e Tecnologia e } \\
\text { Insumos Estratégicos do Ministério da } \\
\text { Saúde. Conselho Consultivo instituído } \\
\text { pela Portaria no. } 2.363 \text {, de } 07 / 10 / 2009^{41}\end{array}$ & $\begin{array}{l}\text { Parceria entre a Secretaria Municipal de Saúde de } \\
\text { Piripiri /Departamento de Ciência e Tecnologia } \\
\text { da Secretaria de Ciência e Tecnologia e Insumos } \\
\text { Estratégicos do Ministério da Saúde/Centro } \\
\text { Latino-Americano e do Caribe de Informação } \\
\text { em Ciências da Saúde/EVIPNet Brasil. Instituído } \\
\text { pela Portaria no }{ }^{\circ} .032 / 10-\text { SMS }^{37} \text { de 22/04/2010 }\end{array}$ \\
\hline Financiamento & $\begin{array}{l}\text { Investimento e custeio por recursos do } \\
\text { Ministério de Saúde }\end{array}$ & $\begin{array}{l}\text { Investimento e custeio pelo Fundo Municipal de } \\
\text { Saúde de Piripiri }\end{array}$ \\
\hline $\begin{array}{l}\text { Principais } \\
\text { produtos e } \\
\text { atividades }\end{array}$ & $\begin{array}{l}\text { Capacitar técnicos e gestores sobre uso } \\
\text { de evidência; Sintetizar evidências para } \\
\text { as necessidades da gestão de saúde, } \\
\text { utilizando as ferramentas SUPPORT; } \\
\text { Organizar Diálogos Deliberativos com } \\
\text { atores sociais envolvidos em problemas } \\
\text { de saúde prioritários, visando à } \\
\text { disseminação de opções de enfrentamento } \\
\text { informadas por evidências }\end{array}$ & $\begin{array}{l}\text { Sintetizar evidências para as necessidades locais } \\
\text { de saúde, utilizando as ferramentas SUPPORT; } \\
\text { Organizar Diálogos Deliberativos com atores } \\
\text { sociais envolvidos em problemas de saúde } \\
\text { prioritários, visando à disseminação de opções } \\
\text { de enfrentamento informadas por evidências; }\end{array}$ \\
\hline $\begin{array}{l}\text { Localização e } \\
\text { contato }\end{array}$ & $\begin{array}{l}\text { Coordenação-Geral de Gestão do } \\
\text { Conhecimento; Departamento de Ciência } \\
\text { e Tecnologia; Secretaria de Ciência, } \\
\text { Tecnologia e Insumos Estratégicos; } \\
\text { Ministério da Saúde; Setor Comercial } \\
\text { Norte, Quadra 02, Projeção C, sala } \\
\text { 03CEP: 70.712-902 - Brasília - DF } \\
\text { Telefone: (61) 3410-4142/4177 } \\
\text { marina.alves@saude.gov.br, } \\
\text { nathanmendes@hotmail.com }\end{array}$ & $\begin{array}{l}\text { Rua João de Freitas, 11, Piripiri, Piauí, CEP } \\
64.260-000 \\
\text { sms@piripiri.pi.gov.br / } \\
\text { evidenciapiripiri@hotmail.com }\end{array}$ \\
\hline
\end{tabular}

Fontes: Decit/SCTIE/MS. 
cas sobre problemas prioritários locais de saúde, como hipertensão arterial, mortalidade infantil, dengue e fixação de profissionais na APS; 3 ) organização de diálogos deliberativos para a disseminação acerca das opções de enfrentamento e suas estratégias de implementação junto aos interessados e afetados pelo conhecimento e decisão dialogados; 4) promoção da cultura e da institucionalização de incorporação da evidência global às práticas locais no âmbito do sistema de saúde.

A gestão local de saúde de Piripiri apropriouse do NEv/EBVS para enfrentar problemas prioritários. As atividades do NEv/EBVS e seus impactos foram monitoradas e avaliadas e seus resultados foram amplamente disseminados local, nacional e internacionalmente ${ }^{38}$. Essa experiência demonstra que iniciativas institucionalizadas e com suporte de redes e plataformas de tradução do conhecimento podem ser um caminho para a disseminação do uso das evidências científicas na tomada de decisão. Dado o reconhecimento nacional e internacional do NEv/EBVS Piripiri, por seu caráter inovador e seu impacto na gestão e na saúde da população assistida ${ }^{37,42}$, a EVIPNet Brasil replicou tal iniciativa em Sobral (CE) e Recife (PE), e planeja fazê-lo também no Rio de Janeiro, Angra dos Reis (RJ) e Distrito Federal (DF).

As políticas de saúde informadas por evidências poderão tornar-se realidade no Brasil a partir de concomitantes estímulos internacionais (como os citados anteriormente), indução do governo federal (como atualmente exercida pela EVIPNet Brasil e seus colaboradores) e, sobretudo, pelo exercício visionário e determinado da autonomia das gestões municipais de saúde (como Piripiri, Sobral, Recife e as três outras jurisdições atualmente interessadas em replicar tal experiência). Para tal, faz-se necessário que os mecanismos de tradução de conhecimento sejam aperfeiçoados pela experimentação, rigoroso monitoramento, avaliação e disseminação local, nacional e internacional. Ademais, é fundamental o desenvolvimento de competências especificas da gestão municipal da saúde, especialmente relacionadas com a capacidade de usar o conhecimento existente para tomar decisões diante de problemas concretos e prioritários, para melhorar os resultados em saúde e aumentar a eficiência do sistema.

Gray $^{43}$ defende que gestores sanitários contemporâneos devam estar aptos a fazer as perguntas certas, compreender e utilizar os critérios de evidência, avaliar a qualidade das evidências e implementar mudanças informadas pelas evidências. Mas esses atributos necessitam de pro- cessos longitudinais de aprendizado institucional sobre como o conhecimento pode ser usado para informar a decisão e, assim, ser útil à obtenção de melhores resultados em saúde.

Dessa forma, dado que a gestão local da saúde no Brasil está responsável pela maior parte da implementação da política de saúde, o contexto internacional e (crescentemente) nacional favoráveis à tradução do conhecimento em ação, articulados com a concepção de que o conhecimento científico pode informar a tomada de decisão local, proporcionando mais condições de enfrentar problemas na implementação das políticas, resultariam essenciais para o exercício da autonomia, inovação e criatividade do nível local da gestão do SUS. O Brasil poderá assim incrementar base mais sólida de esforços para o incentivo e acompanhamento das iniciativas locais inovadoras de tradução do conhecimento, e avaliar impactos sobre os processos de tomada de decisão e seus resultados na qualidade dos serviços e situação de saúde.

\section{Conclusões}

Neste artigo analisamos o processo de descentralização/municipalização da saúde com um olhar que nos permitiu identificar como foram introduzidos os mecanismos indutórios visando assegurar a implantação das políticas do SUS e que influências tiveram nos espaços de autonomia decisória das gestões locais de saúde. Resultou evidente a variação dessa autonomia, a depender do caráter mais ou menos indutor das normas e dispositivos legais em vigor. Basicamente passamos de uma situação de autonomia mais acentuada até a primeira metade do processo de descentralização para uma redução da mesma, na segunda metade. Tal foi influenciado pelo maior comprometimento dos recursos municipais para garantir a viabilidade na execução das políticas formuladas e normatizadas pelo nível federal.

No contexto mais recente, na vigência do COAP e das atuais políticas de regulação e APS vislumbra-se uma possibilidade de ampliação de autonomia que encerra, em si, uma oportunidade para que se expressem inovação e criatividade por parte dos governos locais, na perspectiva de propiciar qualidade à implantação das políticas do SUS.

É possível e necessário experimentar inovação, criatividade e tomada de decisões informadas pelas melhores evidências, visando melhores resultados em saúde. Estratégias adequadas po- 
dem favorecer o potencial inovador e criativo nos processos de gestão, assim como as iniciativas de consolidação de práticas de formulação e implementação de políticas informadas por evidências no Brasil e no mundo.

\section{Colaboradores}

SF Silva concebeu a ideia inicial, redigiu partes e revisou todas as versões do texto. NM Souza contribuiu com a concepção inicial, redigiu partes e revisou todas as versões do texto. JOM Barreto redigiu partes e revisou todas as versões do texto. 


\section{Referências}

1. Brasil. Ministério da Saúde (MS). Cadastro Nacional de Estabelecimentos de Saúde - CNES; 2013. [acessado 2013 abr 22]. Disponível em:http://cnes.datasus.gov. br/Mod_Ind_Esfera.asp?VEstado $=00$

2. Brasil. Ministério da Saúde (MS). Datasus; 2013. [acessado 2013 abr 24]. Disponível em: http://tabnet.datasus.gov.br/cgi/tabcgi.exe?cnes/cnv/leiintbr. def

3. Brasil. Ministério da Saúde (MS). Departamento de Atenção Básica - DAB; 2013. [acessado 2013 abr 22]. Disponível em: http://dab.saude.gov.br/portaldab/historico_cobertura_sf.php

4. Brasil. Ministério da Saúde (MS). Portaria 545, de 20 de maio de 1993. Estabelece normas e procedimentos reguladores do processo de descentralização das ações e serviços de saúde e aprova a NOB 01/93. Diário Oficial da União 1993; 20 maio.

5. Brasil. Ministério da Saúde (MS). Portaria 2.203, de 6 de novembro de 1996: redefine o modelo de gestão do Sistema Único de Saúde e aprova a NOB 01/96. Diário Oficial da União 1996; 6 nov.

6. Brasil. Ministério da Saúde (MS). Portaria 399/GM, de 22 de fevereiro de 2006. Divulga o Pacto pela Saúde 2006 - Consolidação do SUS e aprova as Diretrizes Operacionais do referido Pacto. Diário Oficial da União 2006; 23 fev.

7. Brasil. Presidência da República. Decreto no $7508 /$ Presidência da República/Casa Civil, de 28 de junho de 2011. Regulamenta a Lei no 8.080 , de 19 de setembro de 1990, para dispor sobre a organização do Sistema Único de Saúde - SUS, o planejamento da saúde, a assistência à saúde e a articulação interfederativa, e dá outras providências. Brasília. Diário Oficial da União 2011; 28 jun.

8. Brasil. Constituição da República Federativa do Brasil de 1988. Diário Oficial da União 1988; 5 out.

9. Brasil. Lei 8.080, de 19 de setembro de 1990. Dispõe sobre as condições para a promoção, proteção e recuperação da saúde, a organização e o funcionamento dos serviços correspondentes e dá outras providências. Diário Oficial da União 1990; 20 set.

10. Brasil. Lei No 8.142, de 28 de dezembro de 1990.Dispõe sobre a participação da comunidade na gestão do Sistema Único de Saúde (SUS) e sobre as transferências intergovernamentais de recursos financeiros na área da saúde e dá outras providências. Diário Oficial da União 1990; 31 dez.

11. Almeida C. O SUS que queremos: sistema nacional de saúde ou subsetor público para pobres? Cien Saude Colet 2003; 8(2):346-369.

12. Cohn A. Questionando o consenso sanitário. Cien Saude Colet 2001; 6(2):292-318.

13. Silva SF. Municipalização da saúde e poder local: sujeitos, atores e políticas. São Paulo: Hucitec; 2001.

14. Brasil. Ministério da Saúde (MS). Secretaria Executiva. Departamento de Economia da Saúde, Investimentos e Desenvolvimento. Brasília; 2012. [acessado 2013 abr 25]. Disponível em: http://portalsaude.saude.gov.br/ portalsaude/arquivos/pdf/2012/Set/28/Despesa_total saude.pdf

15. Campos FE, Machado MH, Girardi SN. A fixação de profissionais de saúde em regiões de necessidades. $D i$ vulgação em Saúde para Debate 2009; 44:13-24.
16. Lima LD, Queiroz LFN, Machado CV, Viana ALA. Descentralização e regionalização: dinâmica e condicionantes da implantação do Pacto pela Saúde no Brasil. Cien Saude Colet 2012; 17(7):1903-1914.

17. Arretche M. Financiamento federal e gestão local de políticas sociais: o difícil equilíbrio entre regulação, responsabilidade e autonomia. Cien Saude Colet 2003; 8(2):331-345

18. Brasil. Ministério da Saúde (MS). Secretaria de Atenção à Saúde. Departamento de Atenção Básica. Política Nacional de Atenção Básica. Brasília: MS; 2012. (Série E. Legislação em Saúde)

19. Brasil. Ministério da Saúde (MS). Portaria no 1.654, de 19 de julho de 2011. Institui, no âmbito do Sistema Único de Saúde, o Programa Nacional de Melhoria do Acesso e da Qualidade da Atenção Básica (PMAQ-AB) e o Incentivo Financeiro do PMAQ-AB, denominado Componente de Qualidade do Piso de Atenção Básica Variável - PAB Variável. Diário Oficial da União 2011; 19 jul.

20. Brasil. Ministério da Saúde (MS). Portaria interministerial no 2.087 , de 1 de setembro de 2011. Institui o programa de valorização da atenção básica. Diário Oficial da União 2011; 1 set.

21. Brasil. Ministério da Saúde (MS). Portaria no 1559/ GM, de 1 de agosto de 2008. Institui a política nacional de regulação do Sistema Único de Saúde - SUS. Diário Oficial da União 2008; 1 ago.

22. Brasil. Ministério da Saúde (MS). Portaria no 4.279, de 30 de dezembro de 2010. Estabelece diretrizes para a organização da Rede de Atenção à Saúde no âmbito do Sistema Único de Saúde (SUS). Diário Oficial da União 2010; 30 dez.

23. Berwick DM. Disseminating Innovations in Health Care. JAMA 2003; 289(15):1969-1975.

24. Ruediger MA. Governança democrática na era da informação. Rev Adm Pública 2003; 37(6):1257-1280.

25. Organização das Nações Unidas (ONU). Declaração do milênio 2000. Lisboa: United Nations Information Centre; 2001. (Resolução A/RES/55/2).

26. Rasanathan K. Task Team for the Global Thematic Consultation on Health in the Post-2015 Development Agenda. What do people want for health in the post2015 agenda? The Lancet 2013; 381(9876):1441-1443.

27. Brasil. Ministério da Saúde (MS). Prêmio Pesquisa para o SUS (PPSUS). [acessado 2013 maio 11]. Disponível em: http://portal.saude.gov.br/portal/saude/Gestor/ visualizar texto.cfm?idtxt $=32726$ \&janela $=1$

28. Jurberg C, Humphreys G. Brazil's march towards universal coverage. Bull World Health Organ 2010; 88(9):646-647.

29. World Health Organization (WHO). The World Health Report 2008: Primary Health Care now more than ever. Washington: WHO; 2008.

30. World Health Organization (WHO). 2005 World Health Assembly Resolution 58.34. WHO; 2005. Disponível em: http://www.paho.org/english/gov/ce/ce136-25-e.pdf

31. Pan-American Health Organization (PAHO). Health Agenda for Americas 2008-2017; WHO, PAHO; 2007. [Cited 2011 Mar 29]. Available from: http://www.paho. org/English/DD/PIN/Health_Agenda.pdf 
32. World Health Organization (WHO). World Health Report 2012: No Health Without Research.WHO; 2011. [Cited 2011 Aug 26]. Available from: http://www.who. int/rpc/whr2012/en/index.html

33. McMaster Health Forum. Knowledge transfer key component of health system research program. [Internet]MHF; 2013.[Cited 2013 Apr 17]. Available from: http://healthforum.mcmaster.ca/index.php/about-us/ new-at-the-forum/1-latest-news/299-forum-shares -in-49-million-grant-from-ministry

34. Australia. Ministry of Health. Strategic Review of Health and Medical Research in Australia - Better Health Through Research. Commonwealth of Australia 2013. [Cited 2013 Apr 15]. Available from: http://www.mckeonreview.org.au/downloads/Strategic_Review_of_Health _and_Medical_Research_Feb_2013-Final_Report.pdf

35. Portal EVIPNet Brasil. Notícias.[Cited 2013 Apr 18]. Available from: http://cspace.evipnetbrasil.bvsalud. org/tiki-view_articles.php

36. Barreto J, Souza N, Abdala C, Panisset U, De Melo G, Pinto R. Curso 'Uso de evidências na gestão municipal da saúde': uma experiência pioneira. Revista Brasileira de Medicina de Família e Comunidade 2012; 7(23):122126.

37. Barreto JOM, Souza NM. Avançando no uso de políticas e práticas de saúde informadas por evidências: a experiência de Piripiri-Piauí. Cien Saude Colet 2013; 18(1):25-34.

38. Lavis JN, Oxman AD, Lewin S, Fretheim A: SUPPORT Tools for evidence-informed health Policymaking (STP). Introduction. Tradução para o português da série de artigos publicados na revista Health Research Policyand Systems. [cited 2013 Apr 18]. Available from: http://brasil.evipnet.org

39. Lavis JN, Oxman AD, Lewin S, Fretheim A. SUPPORT Tools for evidence-informed health Policymaking (STP). Introduction. Health Res Policy Syst 2009; 7(Supl. 1):I1.

40. Piripiri. Secretaria Municipal de Saúde. Portaria no 32/2010. Institui o Núcleo de Evidências em Saúde no âmbito da Secretaria Municipal de Saúde de Piripiri-PI. [acessado 2011 mar 29]. Disponível em: http://www. piripiri.pi.gov.br

41. Brasil. Portaria no. 2.363, de 7 de outubro de 2009. Institui e define atribuições do Conselho Consultivo da EVIPNet Brasil. Diário Oficial da União 2009; 8 out.

42. Barreto JOM, Souza NM .Evidence-informed decision making in local level: the knowledge translation experience in health policy implementation of Piripiri, Brazil. In: Second Global Symposium on Health Systems Research, 2012, Beijing. Book of Abstracts - HSR 2012 Beijing, China. Beijing: Peking University Health Sciences Center; 2012.

43. Gray M. Evidence-based healthcare and public health: how to make decisions about health services and public heath. $3^{\text {th }}$ ed. London: Churchill Livingstone; 2009. 